\title{
Another Notch in the mechanism of Barrett oesophagus progression
}

CS

in patients

with Barrett

oesophagus,

there was an

increase in

Notch signal-

ling that was

associated with

progression
A new study has examined the mechanism of progression from Barrett oesophagus to oesophageal adenocarcinoma (EAC) in mouse and human tissues. This work showed an association between increased Notch signalling and decreased goblet cell density that could lead to new therapeutic strategies to prevent EAC in patients with Barrett oesophagus.

Barrett oesophagus, a precursor lesion to EAC associated with reflux, is characterized by the replacement of squamous epithelium in the distal oesophagus by metaplastic columnar-lined oesophagus (CLE) tissue with goblet cell features. However, the mechanisms and functional role underlying the differentiation of goblet cells during the progression of Barrett oesophagus are unclear.

Notch signalling is key in regulating LGR5 ${ }^{+}$stem cells of the intestinal epithelium and determining cell fate, and it is essential for proliferation and differentiation across the gastro-

intestinal tract. This process might be particularly relevant to Barrett oesophagus and progression to EAC as aberrant Notch signalling has been

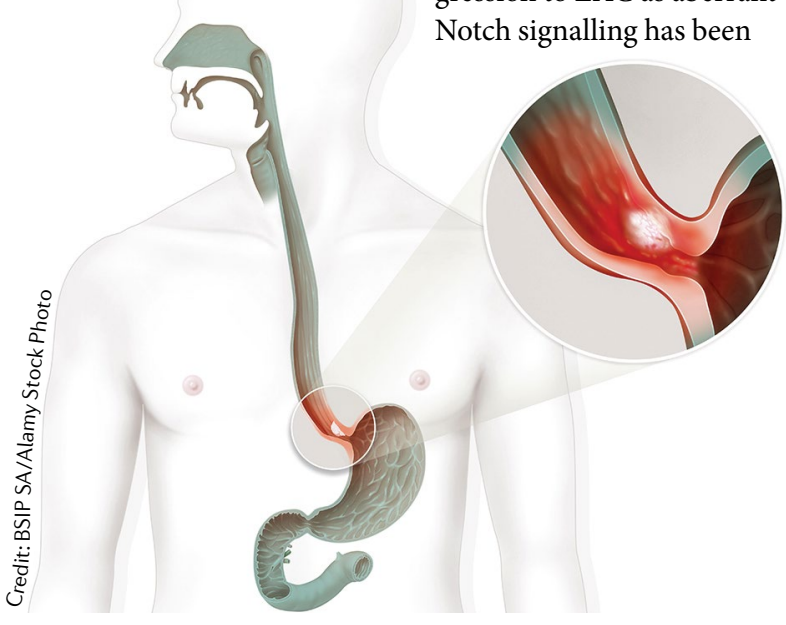

observed in histological specimens from patients with Barrett oesophagus as well as in a transgenic model of Barrett oesophagus (L2-IL1B mice).

"Several reports indicate that Notch signalling is activated in metaplastic Barrett epithelium and inhibition of Notch in animals with reflux results in the conversion of proliferative Barrett oesophagus cells to more differentiated goblet cells," reports corresponding co-author Michael Quante. However, there is also evidence that EAC can arise in a setting of non-metaplastic CLE and in the absence of goblet cells, characterized by high levels of Notch signalling. "These findings have called into question the notion that goblet cell differentiation is strictly associated with cancer risk, and instead raise the interesting hypothesis that goblet cell differentiation and lower levels of Notch signalling in the setting of CLE might be associated with a reduced risk of progression," says corresponding co-author Julian Abrams. "We hypothesized that low levels of Notch might account for a goblet cell-rich phenotype observed in many patients with Barrett oesophagus, and inhibition of Notch signalling might result in terminal differentiation of progenitor cells that otherwise give rise to EAC."

A multicentre study was performed in 164 patients with Barrett oesophagus, with and without dysplasia or early EAC, assessing goblet cell density and aspects of Notch signalling. "In parallel, we performed experiments using the L2-IL1B transgenic mouse model to characterize changes in the Notch pathway, and then examined the effect of Notch acceleration or inhibition," explains Quante.
The researchers found that, in patients with Barrett oesophagus, there was an increase in Notch signalling that was associated with progression to EAC when compared with patients without dysplasia. This upregulation was also inversely correlated with goblet cell density, which was assessed in non-dysplastic regions of the Barrett segment.

In L2-IL1B mice, conditional activation of Notch signalling in LGR5 ${ }^{+}$ progenitor cells reduced the maturation of goblet cells, increased crypt fission and accelerated the development of tumours at the squamocolumnar junction. Conversely, mice with inhibited Notch signalling in LGR5 ${ }^{+}$cells had increased numbers of goblet cells, reduced crypt fission and fewer tumours.

Examining oesophageal tissues from the mice with activated Notch signalling revealed increased levels of Rela, which encodes a subunit of NF- $\kappa \mathrm{B}$, along with evidence of increased NF- $\kappa$ B activity in LGR5 ${ }^{+}$cells. NF- $\kappa B$ signalling sustains a chronic inflammatory microenvironment, and in organoids derived from $L 2-I L 1 B$ mice, the NF- $\kappa B$ inhibitor JSH-23 attenuated Notch-driven tumorigenesis.

"We have demonstrated that increased Notch signalling in Barrett oesophagus decreases goblet cell differentiation and promotes progression to EAC," reports Quante. In previous work, the researchers found that bile acids induce Notch signalling in mice and that gut microbiome alterations occur with progression of Barrett oesophagus. "Thus, we want to further investigate the interaction of bile acids, the microbiome and Notch signalling during oesophageal carcinogenesis," conclude Abrams and Quante.

Iain Dickson

ORIGINAL ARTICLE Kunze, B. et al. Notch signaling mediates differentiation in Barrett's esophagus and promotes progression to adenocarcinoma. Gastroenterology https:// doi.org/10.1053/j.gastro.2020.04.033(2020) 